\title{
VLBA determination of the distance to nearby star-forming regions III. HP Tau/G2 and the three-dimensional structure of Taurus
}

\author{
Rosa M. Torres, Laurent Loinard \\ Centro de Radiostronomía y Astrofísica, Universidad Nacional Autónoma de México, \\ Apartado Postal 72-3 (Xangari), 58089 Morelia, Michoacán, México; \\ r.torres@astrosmo.unam.mx \\ Amy J. Mioduszewski \\ National Radio Astronomy Observatory, Array Operations Center, \\ 1003 Lopezville Road, Socorro, NM 87801, USA \\ and \\ Luis F. Rodríguez \\ Centro de Radiostronomía y Astrofísica, Universidad Nacional Autónoma de México, \\ Apartado Postal 72-3 (Xangari), 58089 Morelia, Michoacán, México
}

\begin{abstract}
Using multi-epoch Very Long Baseline Array observations, we have measured the trigonometric parallax of the weak-line T Tauri star HP Tau/G2 in Taurus. The best fit yields a distance of $161.2 \pm 0.9 \mathrm{pc}$, suggesting that the eastern portion of Taurus (where HP Tau/G2 is located) corresponds to the far side of the complex. Previous VLBA observations have shown that T Tau, to the South of the complex, is at an intermediate distance of about $147 \mathrm{pc}$, whereas the region around L1495 corresponds to the near side at roughly $130 \mathrm{pc}$. Our observations of only four sources are still too coarse to enable a reliable determination of the three-dimensional structure of the entire Taurus star-forming complex. They do demonstrate, however, that VLBA observations of multiple sources in a given star-forming region have the potential not only to provide a very accurate estimate of its mean distance, but also to reveal its internal structure. The proper motion measurements obtained simultaneously with the parallax allowed us to study the kinematics of the young stars in Taurus. Combining the four observations available so far, we estimate the peculiar velocity of Taurus to be about $10.6 \mathrm{~km} \mathrm{~s}^{-1}$ almost completely in a direction parallel to the Galactic plane. Using our improved distance measurement, we have refined the determination of the position on the HR diagram of HP Tau/G2, and of two other members of the HP Tau group (HP Tau itself
\end{abstract}


and HP Tau/G3). Most pre-main sequence evolutionary models predict significantly discrepant ages (by $5 \mathrm{Myr}$ ) for those three stars -expected to be coeval. Only in the models of Palla \& Stahler (1999) do they fall on a single isochrone (at 3 Myr).

Subject headings: Astrometry - Stars: individual HP Tau/G2 - Radio continuum: stars - Radiation mechanisms: non-thermal — Magnetic fields — Stars: formation

\section{Introduction}

Several recent observations (e.g. Loinard et al. 2005, 2007, 2008; Torres et al. 2007; Menten et al. 2007; Xu et al. 2006) have demonstrated that multi-epoch VLBI observations can be used to measure the trigonometric parallax of nearby young stars to better than a few percent. Since the indirect methods traditionally used to estimate the distance to nearby star-forming regions (e.g. Elias 1978a,b, Kenyon et al. 1994, Knude \& Hog 1998) typically have uncertainties of 20\%, VLBI observations have the potential of dramatically improving our knowledge of the space distribution of star-formation around the Sun. With this goal in mind, we have initiated a large project aimed at accurately measuring the trigonometric parallax of a sample of nearby magnetically active young stars using the 10-element Very Long Baseline Array (VLBA) of the National Radio Astronomy Observatory (NRAO). In previous papers of this series, we have reported the distance and proper motions of three young stars in Taurus (T Tauri -Loinard et al. 2007, Hubble 4 and HDE 283572 -Torres et al. 2007). In the present article, we will concentrate on HP Tau/G2, a young star located near the eastern edge of the Taurus complex.

The well-known variable star HP Tau was discovered by Cohen \& Kuhi (1979) to be surrounded by a small group of young stars (called HP Tau/G1, HP Tau/G2, and HP Tau/G3). HP Tau/G1 is located about $20^{\prime \prime}$ north of HP Tau, whereas HP Tau/G2 and HP Tau/G3 are about $15^{\prime \prime}$ to its south-east (see the finding charts in Fig. 22 of Cohen \& Kuhi 1979). HP Tau/G2 and HP Tau/G3 are believed to form a gravitationally bound system with a separation of about $10^{\prime \prime}$. Recently, HP Tau/G3 was itself found to be a tight binary (Richichi et al. 1994), so the HP Tau/G2 - HP Tau/G3 system appears to be a hierarchical triple system. HP Tau/G2 is a weak-line T Tauri star of spectral type G0, with an effective temperature of $6030 \mathrm{~K}$ (Briceño et al. 2002). It is somewhat obscured $\left(A_{V} \sim 2.1 \mathrm{mag}\right.$ ) and has a bolometric luminosity of $6.5 \mathrm{~L}_{\odot}$ (Briceño et al. 2002; Kenyon \& Hartmann 1995). This corresponds to an age of about $10.5 \mathrm{Myr}$ and a mass of $1.58 \mathrm{M}_{\odot}$ (Briceño et al. 2002). The first radio detection of HP Tau/G2 was reported by Bieging et al. (1984) who found a 5 $\mathrm{GHz}$ flux of 5-7 mJy. A few years later, however, the flux had fallen to only about $0.3 \mathrm{mJy}$ (Cohen \& Bieging 1986). Such strong variability is suggestive of non-thermal processes (e.g. Feigelson \& Montmerle 1999). The successful detection of HP Tau/G2 in VLBI experiments (at levels of 1 to $3 \mathrm{mJy}$ ) by Phillips et al. (1991) confirmed the non-thermal origin of the radio emision. 


\section{Observations and data calibration}

In this paper, we will make use of a series of nine continuum $3.6 \mathrm{~cm}(8.42 \mathrm{GHz})$ observations of HP Tau/G2 obtained between September 2005 and December 2007 with the VLBA (Tab. 1). Our phase center was at $\alpha_{J 2000.0}=04^{\mathrm{h}} 35^{\mathrm{m}} 54.161, \delta_{J 2000.0}=+22^{\circ} 54^{\prime} 13^{\prime \prime} \cdot 492$. Each observation consisted of series of cycles with two minutes spent on source, and one minute spent on the main phase-referencing quasar J0426+2327, located 2.14 away. J0426+2327 is a compact ICRF source (Ma et al. 1998) whose absolute position is known to better than 0.7 mas. Every 24 minutes, we also observed two secondary calibrators (J0435+2532 and J0449+1754) which, together with the primary calibrator, form a triangle around the astronomical target (Fig. 1). 
Table 1. Measured source positions and flux densities

\begin{tabular}{|c|c|c|c|c|c|c|c|c|}
\hline \multicolumn{2}{|c|}{ Mean UT date } & Julian Day & $\begin{array}{c}\alpha(\mathrm{J} 2000.0) \\
04^{h} 35^{m}\end{array}$ & $\sigma_{\alpha}$ & $\begin{array}{c}\delta(\mathrm{J} 2000.0) \\
22^{\circ} 54^{\prime}\end{array}$ & $\sigma_{\delta}$ & $\begin{array}{c}f_{\nu} \\
(\mathrm{mJy})\end{array}$ & $\begin{array}{c}\sigma \\
\left(\mathrm{mJy} \mathrm{beam}^{-1}\right)\end{array}$ \\
\hline 2005.09.07 & $12: 36$ & 2453621.02 & $54^{s} 1613574$ & 0.0000034 & $13 " .41131$ & 0.'00009 & 0.71 & 0.06 \\
\hline 2005.11 .16 & 08:01 & 2453690.83 & $54^{s} 1612212$ & 0.0000019 & 13 ".40798 & $0 . .00007$ & 0.97 & 0.07 \\
\hline 2006.01 .23 & $03: 33$ & 2453758.65 & $54^{s} 1609360$ & 0.0000042 & 13 ".40439 & $0 . .00010$ & 0.99 & $0.07 \stackrel{1}{\perp}$ \\
\hline 2006.03.31 & $23: 06$ & 2453826.46 & $54^{s} 1610940$ & 0.0000032 & $13 " .40161$ & 0.00010 & 0.68 & 0.07 \\
\hline 2006.06.10 & $18: 27$ & 2453897.27 & $54^{s} 1617432$ & 0.0000008 & 13 "'39959 & 0.'00002 & 3.06 & 0.08 \\
\hline 2006.09.08 & $12: 33$ & 2453987.02 & $54^{s} 1623557$ & 0.0000024 & $13^{\prime \prime} 39681$ & $0 . .00007$ & 1.08 & 0.06 \\
\hline 2007.06.04 & $18: 56$ & 2454256.29 & $54^{s} 1627018$ & 0.0000038 & $13^{\prime \prime} 38267$ & $0 . .00013$ & 0.63 & 0.07 \\
\hline 2007.09.03 & $12: 53$ & 2454347.04 & $54^{s} 1633509$ & 0.0000025 & $13^{\prime \prime} .38127$ & $0 . .00008$ & 0.78 & 0.06 \\
\hline 2007.12.04 & $06: 51$ & 2454438.79 & $54^{s} 1631378$ & 0.0000037 & 13 "!37605 & 0.'00009 & 0.76 & 0.05 \\
\hline
\end{tabular}


The data were edited and calibrated using the Astronomical Image Processing System (AIPS -Greisen 2003). The basic data reduction followed the standard VLBA procedure for phasereferenced observations, and was described in detail in Loinard et al. (2007). Using the secondary calibrators, we applied the multi-source calibration strategy described in Torres et al. (2007) to correct for systematic errors due to inaccuracies in the troposphere model used, and to clock, antenna, and source position errors. This resulted in significant improvements in the final phase calibration and image quality.

Six hours of telescope time were allocated to each of the first six observations, whereas 9 hours were allocated for each of the last three. Because of the time spent on the calibrators, however, only about 3 and 5 hours were actually spent on source during the first six and the following three observations, respectively. Once calibrated, the visibilities were imaged with a pixel size of 50 $\mu$ as after weights intermediate between natural and uniform (ROBUST $=0$ in AIPS) were applied. This resulted in typical r.m.s. noise levels of $0.06-0.08$ and $0.05-0.07 \mathrm{mJy}$ beam $^{-1}$ during the first six and the last three observations, respectively (Tab. 1). The source was detected with a signal to noise ratio of 10 or better at each epoch (Tab. 1). The source position (also listed in Tab. 1) was determined using a 2D Gaussian fitting procedure (task JMFIT in AIPS). This task provides an estimate of the position error (columns 3 and 5 of Tab. 1) based on the expected theoretical astrometric precision of an interferometer (Condon 1997). However, in spite of the extra calibration steps taken to improve the phase calibration, uncorrected systematic errors still exist, and must be

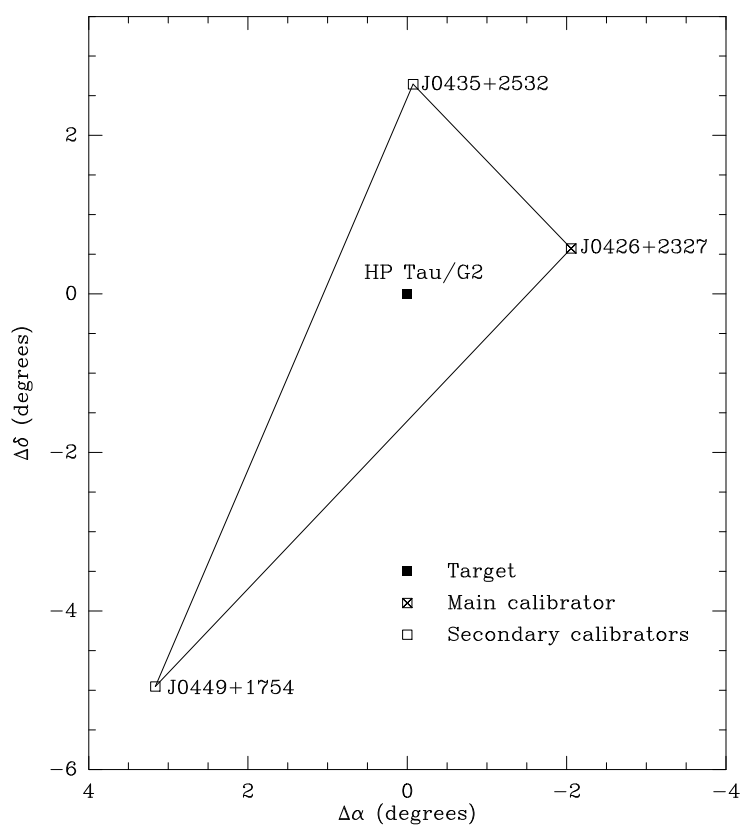

Fig. 1.- Relative position of the astronomical target, the main calibrator $(\mathrm{J} 0426+2327)$, and the secondary calibrators (J0435+2532, and J0449+1754). 


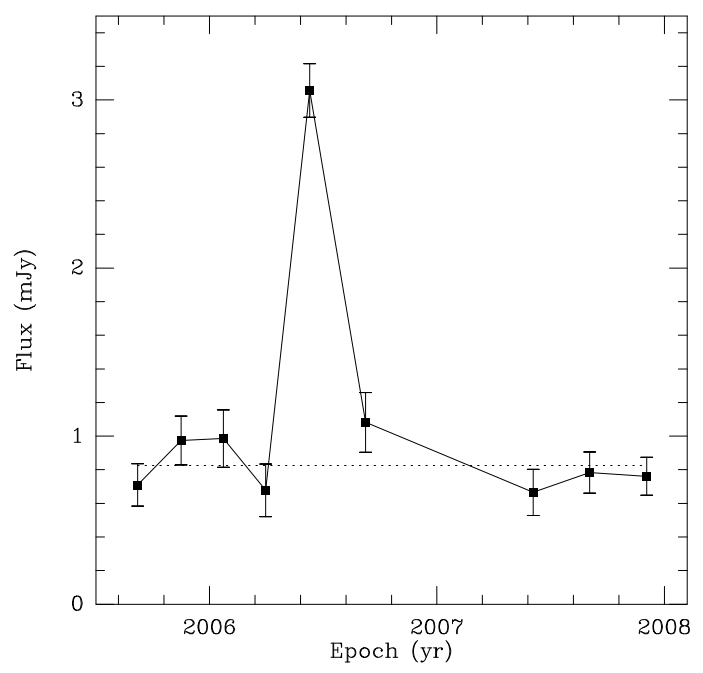

Fig. 2.- Time evolution of the $3.6 \mathrm{~cm}$ flux of HP Tau/G2. Note the flare during the fifth observation.

added quadratically to the values listed in Tab. 1. These remaining systematic errors are difficult to estimate a priori, and may depend on the structure of the source under consideration. Here, we will estimate them from the fits to the data (see below).

\section{Results}

\subsection{Variability and morphological changes}

The flux of HP Tau/G2 was fairly constant around $0.8 \mathrm{mJy}$ at eight of our nine observations. During the fifth observation (June 2006), however, HP Tau/G2 clearly underwent a flaring event, reaching a flux 3 to 4 times higher than that at the other epochs (Fig. 2). This type of variability is not unexpected for non-thermal sources such as HP Tau/G2 (e.g. Feigelson \& Montmerle 1999; Loinard et al. 2008), and is consistent with previous radio measurements (Bieging et al. 1984; Cohen \& Bieging 1986; Phillips et al. 1991; see Sect. 1). The radio source was found to be unresolved at all epochs except the 7th (obtained in June 2007) when it was clearly extended in the north-south direction, with a deconvolved size in that direction of about 2.5 mas (Fig. 3). This increase in the source size might be due to changes in the structure of the active magnetosphere of HP Tau/G2. Interestingly, however, this observation does not correspond to an epoch when the source was particularly bright, nor particularly dim. In any event, the determination of the source position for that epoch is adversely affected by the fact that the source is extended (see below). 

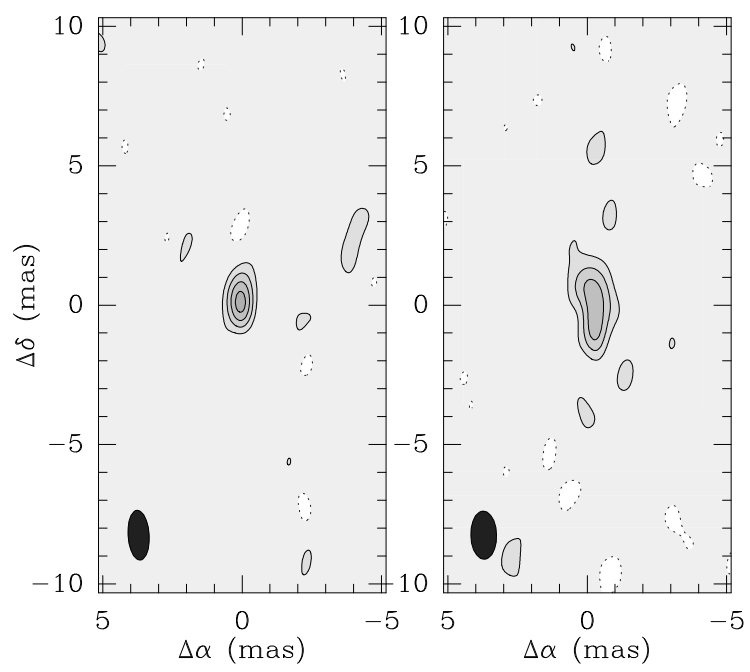

Fig. 3.- Images of HP Tau/G2 at the sixth (left) and seventh (right) epochs. The first contour and the contour spacing in both images is $0.12 \mathrm{mJy} \mathrm{beam}^{-1}$, and the synthesized beams are shown at the bottom left of each panel. Note how the source is elongated in the north-south direction during the seventh epoch, whereas it is unresolved during the sixth observation.

\subsection{Astrometry}

The displacement of HP Tau/G2 on the celestial sphere is the combination of its trigonometric parallax $(\pi)$ and proper motion $(\mu)$. Since HP Tau/G2 is a member of a triple system (see Sect. 1), we should in principle describe its proper motion as the combination of the uniform motion of the center of mass and a Keplerian orbit. This is not necessary, however, because the orbital period of the system must be very much longer than the timespan covered by our observations. If we assume that the total mass of the HP Tau/G2 - HP Tau/G3 system is 2-3 $\mathrm{M}_{\odot}$ and that the current observed separation is a good estimate of the system's semi-major axis, then the orbital period is expected to be 35,000 to 45,000 yr. This is indeed very much longer that the $2 \mathrm{yr}$ covered by our observations, and the acceleration terms can be safely ignored. The astrometric parameters were calculated using the SVD-decomposition fitting scheme described by Loinard et al. (2007). The necessary barycentric coordinates of the Earth, as well as the Julian date of each observation were calculated using the Multi-year Interactive Computer Almanac (MICA) distributed as a CDROM by the US Naval Observatory. The reference epoch was taken at the mean of our observations: JD $2454029.90 \equiv \mathrm{J} 2006.81$.

Since the source was elongated in the north-south direction during the seventh observation, two different fits were made: one where the seventh epoch was included, and one where it was ignored. When the seventh epoch is included, we obtain the following astrometric parameters: 


$$
\begin{aligned}
\alpha_{J 2006.81} & =04^{\mathrm{h}} 35^{\mathrm{m}} 54^{\mathrm{s}} .162033 \pm 0.000003 \\
\delta_{J 2006.81} & =22^{\circ} 54^{\prime} 13^{\prime \prime} .49345 \pm 0^{\prime \prime} 000020 \\
\mu_{\alpha} \cos \delta & =13.90 \pm 0.06 \mathrm{mas} \mathrm{yr}^{-1} \\
\mu_{\delta} & =-15.6 \pm 0.3 \mathrm{mas} \mathrm{yr}^{-1} \\
\pi & =6.19 \pm 0.07 \text { mas. }
\end{aligned}
$$

This corresponds to a distance of $161.6 \pm 1.7 \mathrm{pc}$. The postfit r.m.s. in this case is 0.12 mas in right ascension and 0.51 mas in declination 1 . To obtain a reduced $\chi^{2}$ of one in both right ascension and declination, one must add quadratically $8.8 \mu$ s and 0.59 mas in right ascension and declination, respectively, to the errors listed in Tab. 1. The uncertainties on the parameters quoted above include these systematic contributions. Note that the seventh epoch contributes significantly to the total post-fit r.m.s. since the position corresponding to that observation is farther from the fit (both in right ascension and declination) than that at any other epoch (Fig. 4). If the seventh observation is ignored, the best fit yields the following parameters:

$$
\begin{aligned}
\alpha_{J 2006.81} & =04^{\mathrm{h}} 35^{\mathrm{m}} 54^{\mathrm{s}} .162030 \pm 0.000002 \\
\delta_{J 2006.81} & =22^{\circ} 54^{\prime} 13^{\prime \prime} .49362 \pm 0^{\prime \prime} 000014 \\
\mu_{\alpha} \cos \delta & =13.85 \pm 0.03 \text { mas yr}^{-1} \\
\mu_{\delta} & =-15.4 \pm 0.2 \text { mas yr }^{-1} \\
\pi & =6.20 \pm 0.03 \text { mas. }
\end{aligned}
$$

All these parameters are consistent within $1 \sigma$ with those obtained when the seventh observation is included. The corresponding distance in this case is $161.2 \pm 0.9 \mathrm{pc}$, and the post-fit r.m.s. is 0.058 mas in right ascension and 0.33 mas in declination, significantly better than in the previous fit. Indeed, to obtain a reduced $\chi^{2}$ of one in both right ascension and declination, one must only add quadratically $3.65 \mu$ s and 0.38 mas to the formal errors delivered by JMFIT. Again, the uncertainties on the parameters quoted above include these systematic contributions.

As mentioned earlier, the source during the seventh epoch was extended, and the astrometry consequently less reliable. Since the fit when it is ignored is clearly much better than that when it is included, we consider the second fit above our best result.

\footnotetext{
${ }^{1}$ The residual is much larger in declination than in right ascension. We will come back to this point in Sect. 3.3.
} 


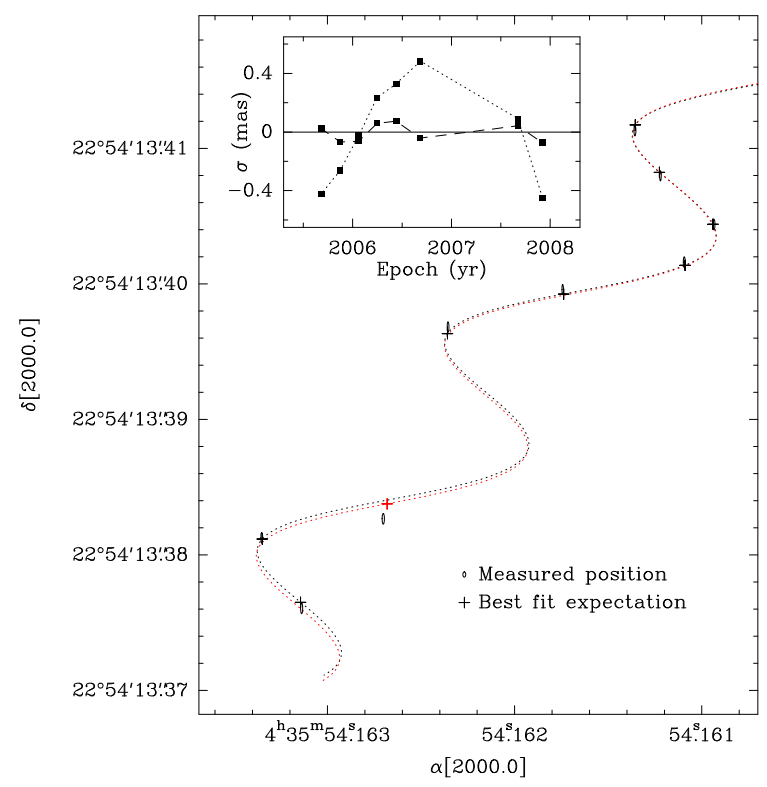

Fig. 4.- Measured positions and best fits for HP Tau/G2. The observed positions are shown as ellipses, the size of which represents the error bars. Two fits are shown: the dotted black line corresponds to the fit where the 7th epoch is ignored, whereas the dotted red line is the fit where it is included. Note that the 7 th observation falls significantly to the south of either fit. The inset shows the fit residuals (of the fit without the 7th epoch) in right ascension (dashed line) and declination (dotted line). Note the large residuals in declination.

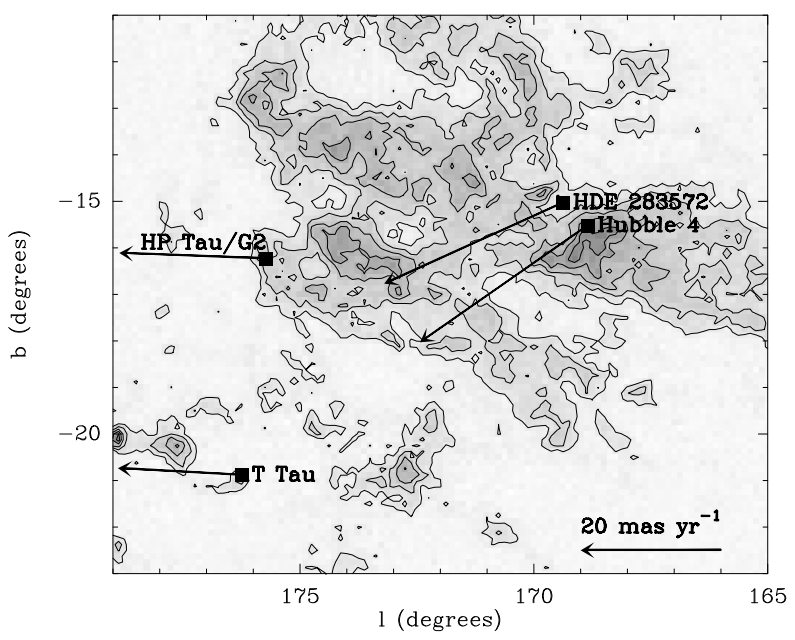

Fig. 5.- Positions and proper motions of Hubble 4, HDE 283572, T Tau, and HP Tau/G2 superposed on the CO(1-0) map of Taurus from Dame et al. (2001). 


\subsection{Error analysis}

It is noteworthy that, whether or not the seventh epoch is included, the post-fit r.m.s. and the systematic error contribution that must be added to the uncertainties quoted in Tab. 1, are much larger in declination than in right ascension. Fortunately, this large declination contribution does not strongly affect the distance determination, because the strongest constraints on the parallax come from the right ascension measurements. Interestingly, this is, with Hubble 4 (Torres et al. 2007), the second source for which we find large systematic declination residuals. Astrometric fitting of phase-referenced VLBI observations is usually worse in declination than in right ascension (e.g. Fig. 1 in Chatterjee et al. 2004) as a result of residual zenith phase delay errors (Reid et al. 1999). In the case of Hubble 4, however, we argued that the large post-fit declination r.m.s. might trace the reflex motion caused by an unseen companion, because a periodicity of about 1.2 yr could be discerned in the residuals. In the present source, the case for a periodicity is less clear (Fig. 4, inset), but the residuals are clearly not random. Interestingly, the large residuals are in the same north-south direction as the extension of the source seen during our 7th observation. This orientation might, therefore, correspond to a preferred direction of the system along which it tends to vary more strongly. Additional observations will clearly be necessary to settle this issue. 
Table 2: Radial velocities, proper motions, heliocentric, and peculiar velocities in Galactic coordinates for the 4 sources observed with the VLBA so far.

\begin{tabular}{|c|c|c|c|c|c|c|c|c|c|c|}
\hline Source & $\begin{array}{c}V_{r} \\
\left(\mathrm{~km} \mathrm{~s}^{-1}\right)\end{array}$ & $\begin{array}{r}\mu_{\ell} \cos (b) \\
(\text { mas }\end{array}$ & $r^{-1}{ } \mu_{b}$ & $U$ & $\begin{array}{c}V \\
\left(\mathrm{~km} \mathrm{~s}^{-1}\right)\end{array}$ & $W$ & $u$ & $\begin{array}{c}v \\
\left(\mathrm{~km} \mathrm{~s}^{-1}\right)\end{array}$ & $w$ & References $^{\mathrm{a}}$ \\
\hline HP Tau/G2 & $17.7 \pm 1.8$ & $+20.90 \pm 0.07$ & $+0.82 \pm 0.10$ & -18.59 & -14.65 & -4.50 & -8.59 & -9.40 & +2.67 & 1,2 \\
\hline Hubble 4 & $15.0 \pm 1.7$ & $+23.94 \pm 0.12$ & $-16.74 \pm 0.15$ & -14.96 & -12.66 & -14.30 & -4.96 & -7.41 & -7.13 & 3,4 \\
\hline HDE 283572 & $15.0 \pm 1.5$ & $+25.53 \pm 0.05$ & $-11.61 \pm 0.06$ & -15.89 & -13.07 & -10.84 & -5.89 & -7.82 & -3.67 & 3,2 \\
\hline $\mathrm{T} \mathrm{Tau}^{\mathrm{b}}$ & $19.1 \pm 1.2$ & $+17.76 \pm 0.03$ & $+0.99 \pm 0.04$ & -19.09 & -11.27 & -6.30 & -9.09 & -6.02 & +0.87 & 4,5 \\
\hline
\end{tabular}

${ }^{a}{ }_{1}=$ This work; $2=$ Walter et al. 1988; 3=Torres et al. 2007; 4=Hartmann et al. 1986; 5=Loinard et al. 2007

${ }^{b}$ The radial velocity and proper motions used here are those of $\mathrm{T}$ Tau N. The radial velocities for T Tau Sa and T Tau

$\mathrm{Sb}$ are available in Duchêne et al. (2002) and are very similar. 


\section{Discussion}

\subsection{Kinematics of the sources in Taurus}

For Galactic sources, it is interesting to express the proper motions in Galactic coordinates rather than in the equatorial system naturally delivered by the VLBA. The results for HP Tau, and the three sources previously observed with the VLBA are given in columns 3 and 4 of Tab. 2. Interestingly, the proper motion of HP Tau/G2 is very similar to that of $\mathrm{T}$ Tau, but significantly different from those of Hubble 4 and HDE 283572 (which are themselves very similar to each other). HP Tau/G2 and T Tau also happen to both be located on the eastern side of the Taurus complex, whereas Hubble 4 and HDE 283572 are both around Lynds 1495 near the center of the complex (Fig. 5).

There is a fifth star in Taurus (V773 Tau) with VLBI-based proper motion and trigonometric parallax measurements (Lestrade et al. 1999). V773 Tau is located about a degree south-west of Hubble 4, and the proper motions reported by Lestrade et al. (1999) are similar to those of Hubble 4 and HDE 283572 (see Fig. 4 in Torres et al. 2007). It is now known that V773 Tau is a quadruple system composed of a tight spectroscopic binary orbited by two companions. The radio source observed by Lestrade et al. (1999) is associated with the spectroscopic binary (the other two stars do not appear to be detectable radio emitters). In several VLBI observations (e.g. Phillips et al. 1991, Boden et al. 2007, Torres et al. 2008), the radio source has been reported to be double, with each component tracing one of the stars in the spectroscopic binary. This binarity was not taken into account by Lestrade et al. (1999), and may have affected their parallax measurement. Indeed, Boden et al. (2007) recently modeled the orbit of V773 Tau combining spectroscopic observations, Keck Interferometer data and VLBA imaging. The distance to V773 Tau that they obtain (136.2 $\pm 3.7 \mathrm{pc})$ is somewhat smaller than the value $\left(148.4_{-5.3}^{+5.7} \mathrm{pc}\right)$ reported by Lestrade et al. (1999). Because of this slight discrepancy, we will not include V773 Tau in the present analysis. It should be mentioned that we are currently analyzing new multi-epoch VLBA observations of V773 Tau designed to constrain both its distance and orbital motions. These data will be published in a forthcoming paper.

Knowing the distance to the sources with high accuracy, it is possible to transform the observed proper motions into transverse velocities. Combining this information with radial (Heliocentric) velocities taken from the literature (second column of Tab. 2), it becomes possible to construct the three-dimensional velocity vectors. It is common to express these vectors on a rectangular $(X, Y, Z)$ coordinate system centered in the Sun, with $X$ pointing towards the Galactic center, $Y$ in the direction of Galactic rotation, and $Z$ towards the Galactic North Pole. In this system, the coordinates of the Heliocentric velocities will be written $(U, V, W)$. As a final step, it is also possible to calculate the peculiar velocity of the stars. This involves two stages: first, the peculiar 
motion of the Sun must be removed to transform the Heliocentric velocities into velocities relative to the LSR. Following Dehnen \& Binney (1998), we will use $u_{0}=+10.00 \mathrm{~km} \mathrm{~s}^{-1}, v_{0}=+5.25 \mathrm{~km}$ $\mathrm{s}^{-1}$, and $w_{0}=+7.17 \mathrm{~km} \mathrm{~s}^{-1}$ for the peculiar velocity of the Sun expressed in the coordinate system defined above. The second stage consists in estimating the difference in circular velocity between Taurus and the Sun, so the peculiar velocities are expressed relative to the LSR appropriate for Taurus, rather than relative to the LSR of the Sun. This was done assuming the rotation curve of Brand \& Blitz (1993), and represents a small correction of only about $0.3 \mathrm{~km} \mathrm{~s}^{-1}$. We will write $(u, v, w)$ the coordinates of the peculiar velocity of the sources. Both $(U, V, W)$ and $(u, v, w)$ are given in Tab. 2 for the four sources considered here. Their projections onto the $(X, Y),(X, Z)$, and $(Y, Z)$ planes are shown in Fig. 6.

The mean heliocentric velocity and the velocity dispersion of the four sources are:

$$
\begin{aligned}
U & =-17.1 \pm 1.7 \mathrm{~km} \mathrm{~s}^{-1} \\
V & =-12.9 \pm 1.2 \mathrm{~km} \mathrm{~s}^{-1} \\
W & =-9.0 \pm 3.8 \mathrm{~km} \mathrm{~s}^{-1}
\end{aligned}
$$

These values are similar to those reported by Bertout \& Genova (2006) for a larger sample of young stars in Taurus with optically measured proper motions. Note that the velocity dispersion in the $W$ direction is somewhat artificially high because (as noted earlier) Hubble 4 and HDE 283572 on the one hand, and T Tau and HP Tau/G2 on the other, clearly have different vertical velocities. They likely belong to two different kinematic sub-groups.

The mean peculiar velocity of the four sources considered here is:

$$
\begin{aligned}
u & =-7.1 \pm 1.7 \mathrm{~km} \mathrm{~s}^{-1} \\
v & =-7.7 \pm 1.2 \mathrm{~km} \mathrm{~s}^{-1} \\
w & =-1.8 \pm 3.8 \mathrm{~km} \mathrm{~s}^{-1}
\end{aligned}
$$

We argue that this is a good estimate of the mean peculiar velocity of the Taurus complex. This velocity is almost entirely in the $(X, Y)$ plane. Thus, although Taurus is located significantly out of the mid-plane of the Galaxy (about $40 \mathrm{pc}$ to its south), it appears to be moving very little in the vertical direction. The motion in the $(X, Y)$ plane, on the other hand, is fairly large, leading to a total peculiar velocity $\left(u^{2}+v^{2}+w^{2}\right)^{0.5}=10.6 \mathrm{~km} \mathrm{~s}^{-1}$. According to Stark \& Brand (1989), the one-dimensional velocity dispersion of giant molecular clouds within $3 \mathrm{kpc}$ of the Sun is about 8 $\mathrm{km} \mathrm{s}^{-1}$. As a consequence, each component of the peculiar velocity of a given molecular cloud 

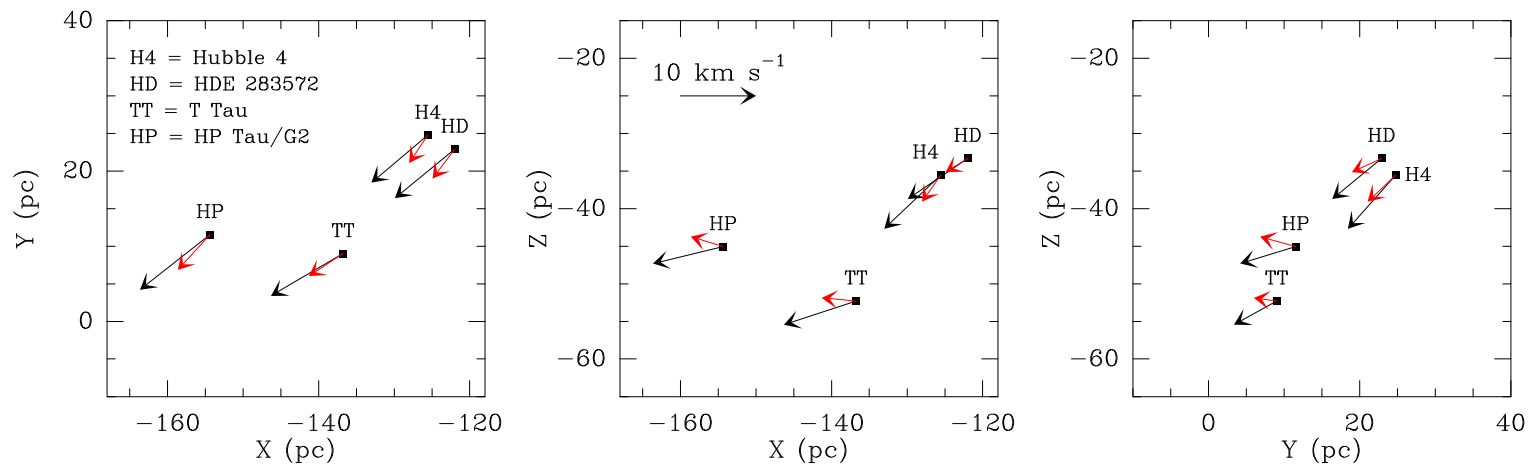

Fig. 6.- Heliocentric velocities (black arrows) and peculiar velocities (red arrows) for the four stars in Taurus with VLBA-based distance determinations.

is expected to be of that order, and our determination of the mean peculiar velocity of Taurus is in reasonable agreement with that prediction. Another useful comparison is with the velocity dispersion of young main sequence stars. For the bluest stars in their sample (corresponding to early A stars), Dehnen \& Binney (1998) found velocity dispersions of about $6 \mathrm{~km} \mathrm{~s}^{-1}$ in the vertical direction, and of $10-14 \mathrm{~km} \mathrm{~s}^{-1}$ in the $X$ and $Y$ directions. The young stars in Taurus are significantly younger than typical main sequence early A stars, so one would expect young stars in Taurus to have peculiar velocities somewhat smaller than $6 \mathrm{~km} \mathrm{~s}^{-1}$ in the vertical direction, and than $10-14 \mathrm{~km} \mathrm{~s}^{-1}$ in the $X$ and $Y$ directions. This is indeed what is observed. Note, however, that Taurus is not among the star-forming regions with the smallest peculiar velocities. In Orion, Gómez et al. (2005) found a difference between expected and observed proper motions smaller than $0.5 \mathrm{~km} \mathrm{~s}^{-1}$.

One last comment should be made here. The data presented here and in the other papers of this series yield proper motions and trigonometric parallaxes that, together, enable the measurement of transverse velocities with an accuracy of about $1 \%$. For sources in Taurus, this corresponds to an absolute error better than $0.1 \mathrm{~km} \mathrm{~s}^{-1}$. In comparison, the radial velocity measurements available in the literature have typical uncertainties of 1 to $2 \mathrm{~km} \mathrm{~s}^{-1}$. To take full advantage of the VLBA data, it will become important to measure radial velocities with a significantly improved accuracy.

\subsection{Distance and structure of the Taurus association}

Taking the mean of the four VLBA-based parallax measurements available (HP Tau/G2, T Tau, Hubble 4 and HDE 283572), we can estimate the mean parallax to the Taurus complex to be $\bar{\pi}=7.08$ mas. This corresponds to a mean distance $\bar{d}$ of $141.2 \mathrm{pc}$, in good agreement with previous estimates (Kenyon et al. 1994). 
The angular size of Taurus is about $10^{\circ}$, corresponding to a physical size of roughly $25 \mathrm{pc}$. It would be natural to expect that the depth of Taurus might be similar, and that different sources may be found at significantly different distances from us. The observations presented here and in the previous papers of this series are, however, the first ones with enough accuracy to directly probe the depth of the Taurus complex. They reveal that HP Tau is about $30 \mathrm{pc}$ farther than Hubble 4 and HDE 283572, and that Taurus is at least as deep as it is wide. A trivial but important consequence is that using the mean distance indiscriminately for all the stars in the complex will result in systematic errors at the levels of about $10 \%$. To reach higher accuracy, one will have to reconstruct the complete three-dimensional structure of Taurus. The number of sources considered so far is obviously too limited to obtain such a complete view. It is interesting to note, however, that Hubble 4 and HDE 283572 which are very near one another in projection, and share the same kinematics (See Sect. 4.2), are also found to be at similar distances from us ( $\sim 130 \mathrm{pc})$. This suggests that there exist in that region (corresponding to the surroundings of the dark cloud Lynds 1495) a coherent spatio-kinematical structure at about $130 \mathrm{pc}$. Observations with an astrometric precision similar to that of the data presented here for several dozen young stars would allow the identification of several such coherent groups across the complex. This, in turn, would allow a fairly accurate re-construction of a three-dimensional structure of Taurus. Currently, Very Long Baseline Interferometry is the only technique with sufficient accuracy to carry out the necessary observations.

\subsection{Comparison with theoretical evolutionary tracks}

As mentioned in Sect. 1, HP Tau/G2 is a member of a compact group of four young stars, comprising HP Tau itself, HP Tau/G1, G2, and G3. Given the small angular separations between them, the members of this group are very likely to be physically associated -indeed, HP Tau/G2 and G3 are thought to form a bound system. They are, therefore, very likely to be at the same distance from the Sun. Using our accurate estimate of the distance to HP Tau/G2, we are now in a position to refine the determination of the luminosities of all four stars. Little is known about HP $\mathrm{Tau} / \mathrm{G} 1$, but the effective temperature and the bolometric luminosity (obtained assuming $d=142$ pc) of the other three members are given in Briceño et al. (2002). Those values (corrected to the new distance) allow us to place the stars accurately on an HR diagram (Fig. 7).

From their position on the HR diagram, one can (at least in principle) derive the mass and age of the stars using theoretical pre-main sequence evolutionary codes. Several such models are available, and we will use four of them here2: those of Siess et al. (2000), Demarque et al. (2004;

\footnotetext{
${ }^{2}$ The models by Baraffe et al. (1998) will not be used because they do not cover the mass range of our stars.
} 

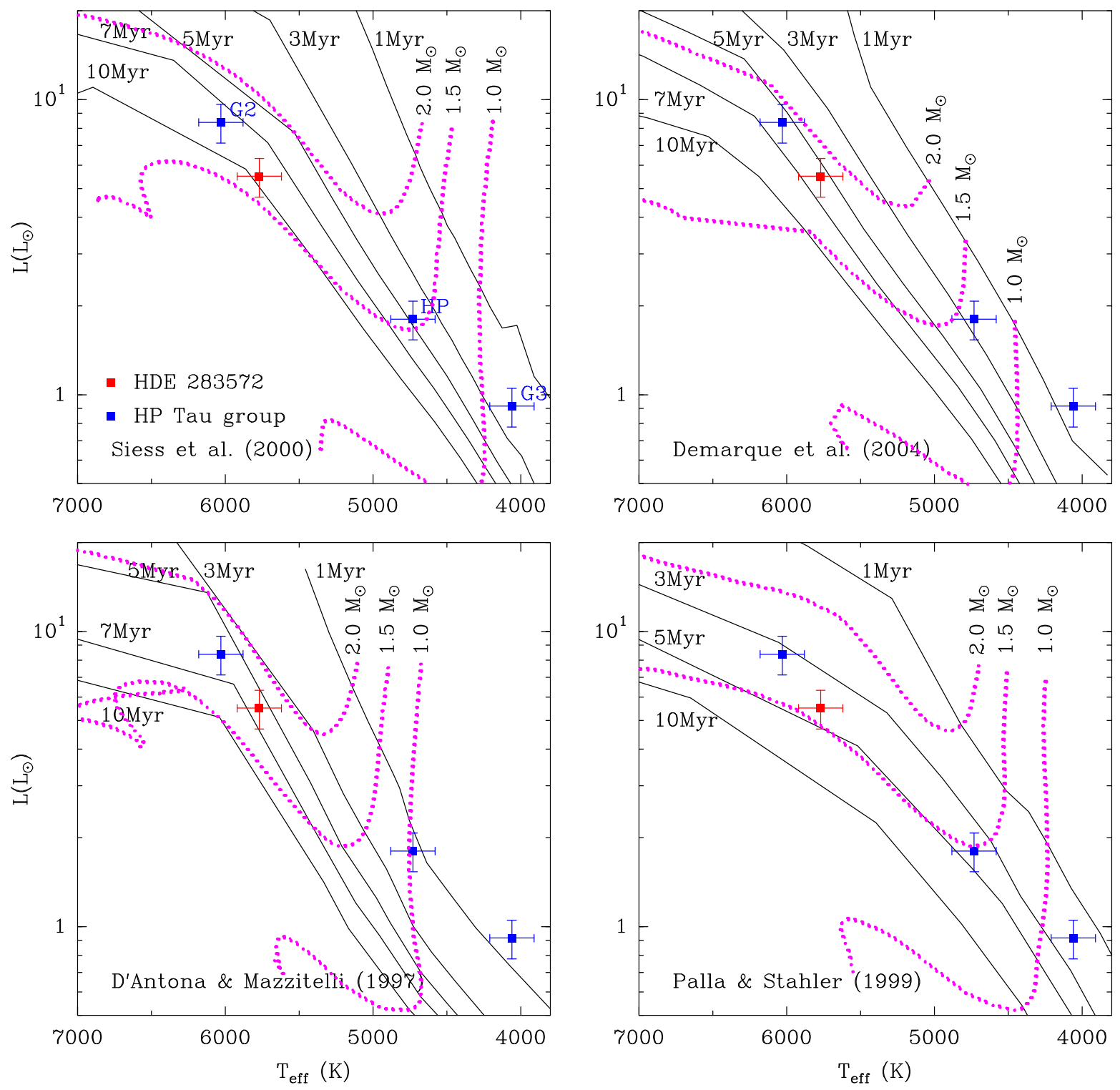

Fig. 7.- Positions of the three HP Tau members (blue symbols) and of HDE 283572 (red symbol) on an HR diagram. (From the coolest to the warmest, the three stars in the HP Tau group are HP Tau/G3, HP Tau, and HP Tau/G2, as indicated in the first panel.) Isochrones (full back lines) are shown at 1, 3, 5, 7, and 10 Myr for various models. For the same models, evolutionary tracks for stars of $1.0,1.5$, and $2.0 \mathrm{M}_{\odot}$ are also shown as dotted magenta lines.

known as the Yonsei-Yale $Y^{2}$ models), D'Antona \& Mazzitelli (1997), and Palla \& Stahler (1999). The isochrones for those four models at 1, 3, 5, 7, and $10 \mathrm{Myr}$ are shown as solid black lines in Fig. 7. Also shown are the evolutionary tracks (from the same models) for stars of 1.0, 1.5, and $2.0 \mathrm{M}_{\odot}$. The three HP Tau members are shown as blue symbols, and HDE 283572 (from Torres et 
al. 2007) is shown as a red symbol. (We will discuss momentarily the reason for incorporating that source in the present analysis.)

A number of interesting points can be seen from Fig. 7. First, there is reasonable agreement (within $40 \%$, see below) between the masses predicted by different models. The best case is that of HP Tau/G2, for which the different models predict masses consistent with each other at the $10 \%$ level (between 1.7 and $1.9 \mathrm{M}_{\odot}$ ). The situation for HP Tau is somewhat less favorable, since the models of Siess et al. (2000) or Palla \& Stahler (1999) predict a mass of $\sim 1.5 \mathrm{M}_{\odot}$, whereas those of D'Antona \& Mazzitelli (1997) predicts a significantly smaller mass of $\sim 1.0$ $\mathrm{M}_{\odot}$. Thus, there is a 35\% spread in the values predicted by different models for the mass of that source. The least favorable situation is for HP Tau/G3. The mass of that source is about $0.8 \mathrm{M}_{\odot}$ according to the models of Siess et al. (2000), but slightly less than $0.5 \mathrm{M}_{\odot}$ according to those D'Antona \& Mazzitelli (1997). This is a 40\% discrepancy. This tendency for pre-main sequence evolutionary models to become more discrepant at lower mass had been noticed before, and is discussed at length in Hillenbrand et al. (2008). In the absence of dynamically measured masses, it is impossible to assess which of the models used here provides the "best" answer.

Another interesting issue is related to the age predictions of the different models. Since the different members of the HP Tau group are likely to be physically associated, they are expected to be nearly coeval. This is particularly true of HP Tau/G2 and HP Tau/G3 which are believed to form a loose binary system. Interestingly, most models predict significantly different ages for the three sources (see Fig. 7). The models by Siess et al. (2000) predict ages of about $8 \mathrm{Myr}$ and $3 \mathrm{Myr}$ for HP Tau/G2 and HP Tau/G3, respectively. A similar 5 Myr age difference is found for the models of Demarque et al. (2004) and D'Antona \& Mazzitelli (1997): both predict ages slightly smaller than $1 \mathrm{Myr}$ for HP Tau/G3, and somewhat larger than $5 \mathrm{Myr}$ for HP Tau/G2. In principle, those differences could be real. In should be noticed, however, that the vast majority of low-mass stars in Taurus (with spectral types M and late K) have ages smaller than 3 Myr (Briceño et al. 2002). Moreover, mass-dependent systematic effects in the age predictions made by evolutionary tracks have been reported before. In particular, Hillenbrand et al. (2008) argued that existing models could significantly over-predict the age of relatively massive stars $\left(\mathrm{M} \gtrsim 1.5 \mathrm{M}_{\odot}\right)$. HP Tau/G2 is precisely such a fairly massive star. So is HDE 283572, another young star in Taurus with a recently measured accurate distance (Torres et al. 2007). The age estimate for that star based on the models by Siess et al. (2000), Demarque et al. (2004) and D'Antona \& Mazzitelli (1997) is 6$10 \mathrm{Myr}$ (Fig. 7), somewhat larger than would be expected for Taurus. The only of the four models considered here to predict similar ages for the three members of the HP Tau group is that of Palla \& Stahler (1999). Within the errors, all three stars fall on the 3 Myr isochrone. Note that this value is also consistent with the ages of lower mass stars in Taurus (Briceño et al. 2002, see above). 


\section{Conclusions and perspectives}

In this article, we have reported multi-epoch phase-referenced VLBA observations of the weak-line T Tauri star HP Tau/G2 located near the eastern edge of the Taurus star-forming complex. These observations allowed us to measure the trigonometric parallax of the target with an accuracy better than $1 \%$, and to refine the determination of the intrinsic parameters of the source. Combined with previous similar results on other young stars of Taurus, these data also enabled us to probe directly for the first time the depth of this important region of star-formation. We found that HP Tau/G2 is about 30 pc farther than two stars (Hubble 4 and HDE 283572) located close to the dark cloud Lynds 1495, near the central portion of Taurus. This implies that the Taurus complex is at least as deep as it is wide on the plane of the sky. The famous young star T Tauri, located to the south of the complex happens to be at an intermediate distance.

Our observations also allow us to determine the full velocity vector of our sources with excellent accuracy. Combining the results from the four stars considered so far, we estimate the mean peculiar velocity of Taurus to be about $10.6 \mathrm{~km} \mathrm{~s}^{-1}$, oriented almost entirely along the direction of the Galactic plane. The lack of a significant vertical component may appear somewhat surprising given the location of Taurus about $40 \mathrm{pc}$ below the Galactic mid-plane. This might suggest that Taurus has reached its farthest distance from the mid-plane and is about to fall back towards it. Overall, the peculiar velocity of Taurus appears to be in reasonable agreement with measurements of the velocity dispersion of giant molecular clouds and young main sequence stars in the Solar neighborhood.

Using our improved distances, we have refined the determination of the location of the stars in the HP Tau group on an HR diagram, and compared those positions with theoretical models available in the literature. There is reasonable agreement (within 40\%) between the different models on the mass of the stars. It is noteworthy, however, that this agreement becomes progressively poorer as one considers less massive stars. Although the different members of the HP Tau group might be expected to be coeval, three of the theoretical models considered here predict significantly different ages for the various members. Moreover, those models predict ages for the most massive member of the group (HP Tau/G2) somewhat larger than would expected for Taurus. The only model for which all three stars in the HP Tau group fall on a single isochrone ( $3 \mathrm{Myr}$ ) is that of Palla \& Stahler (1999). Similar studies of multiple young stellar systems would clearly help test and improve pre main sequence evolutionary models.

R.M.T., L.L. and L.F.R. acknowledge the financial support of DGAPA, UNAM and CONACyT, México. We are indebted to Tom Dame for sending us a digital version of the integrated $\mathrm{CO}(1-0)$ map of Taurus, to Andy Boden for his help with the PMS models, and to Cesar Briceño

for his detailed comments of the errors affecting the determination of the luminosity and effective 
temperature of the young stars in the HP Tau group. We are also grateful to the anonymous referee, in particular for his/her comments which prompted us to expand significantly our discussion of the comparison with PMS models. The National Radio Astronomy Observatory is a facility of the National Science Foundation operated under cooperative agreement by Associated Universities, Inc.

\section{REFERENCES}

Bertout, C., \& Genova, F. 2006, A\&A, 460, 499

Bieging, J. H., Cohen, M., \& Schwartz, P. R. 1984, ApJ, 282, 699

Boden, A. F., et al. 2007, ApJ, 670, 1214

Brand, J., \& Blitz, L. 1993, A\&A, 275, 67

Briceño, C., Luhman, K. L., Hartmann, L., Stauffer, J. R., \& Kirkpatrick, J. D. 2002, ApJ, 580, 317

Chatterjee, S., Cordes, J. M., Vlemmings, W. H. T., Arzoumanian, Z., Goss, W. M., \& Lazio, T. J. W. 2004, ApJ, 604, 339

Cohen, M., \& Bieging, J. H. 1986, AJ, 92, 1396

Cohen, M., \& Kuhi, L. V. 1979, ApJS, 41, 743

Condon, J. J. 1997, PASP, 109, 166

Dame, T. M., Hartmann, D., \& Thaddeus, P. 2001, ApJ, 547, 792

D’Antona, F., \& Mazzitelli, I. 1997, Memorie della Societa Astronomica Italiana, 68, 807

Demarque, P., Woo, J.-H., Kim, Y.-C., \& Yi, S. K. 2004, ApJS, 155, 667

Dehnen, W., \& Binney, J. J. 1998, MNRAS, 298, 387

Duchêne, G., Ghez, A. M., \& McCabe, C. 2002, ApJ, 568, 771

Elias, J. H. 1978a, ApJ, 224, 857

Elias, J. H. 1978b, ApJ, 224, 453

Feigelson, E.D., \& Montmerle, T., 1999, ARAA, 37, 363 
Gómez, L., Rodríguez, L. F., Loinard, L., Lizano, S., Poveda, A., \& Allen, C. 2005, ApJ, 63

Greisen, E.W. 2003, in Information Handling in Astronomy - Historical Vistas, ed. A. Heck (Dordrecht: Kluwer Academic Publishers), 109

Hartmann, L., Hewett, R., Stahler, S., \& Mathieu, R. D. 1986, ApJ, 309, 275

Hillenbrand, L.A., Bauermeister, A., \& White, R.J. 2008, in 14th Cambridge Workshop on Cool Stars, Stellar Systems, and the Sun ASP Conference Series, Edts: G. Van Belle, 384, 200

Kenyon, S. J., Dobrzycka, D., \& Hartmann, L. 1994, AJ, 108, 1872

Kenyon, S. J., \& Hartmann, L. 1995, ApJS, 101, 117

Knude, J., \& Hog, E. 1998, A\&A, 338, 897

Lestrade, J.-F., Preston, R. A., Jones, D. L., Phillips, R. B., Rogers, A. E. E., Titus, M. A., Rioja, M. J., \& Gabuzda, D. C. 1999, A\&A, 344, 1014

Loinard, L., Mioduszewski, A. J., Rodríguez, L. F., González, R. A., Rodríguez, M. I., \& Torres, R. M. 2005, ApJ, 619, L179

Loinard, L., Torres, R. M., Mioduszewski, A. J., Rodríguez, L. F., González, R. A., Lachaume, R., Vázquez, V., \& González, E. 2007, ApJ, 671, 546

Loinard, L., Torres, R. M., Mioduszewski, A. J., \& Rodríguez, L. F. 2008, ApJ, 675, L29

Ma, C., et al. 1998, AJ, 116, 516

Menten, K. M., Reid, M. J., Forbrich, J., \& Brunthaler, A. 2007, A\&A, 474, 515

Palla, F., \& Stahler, S. W. 1999, ApJ, 525, 772

Phillips, R. B., Lonsdale, C. J., \& Feigelson, E. D., 1991, 382, 261

Reid, M. J., Readhead, A. C. S., Vermeulen, R. C., \& Treuhaft, R. N. 1999, ApJ, 524, 816

Richichi, A., Leinert, C., Jameson, R., \& Zinnecker, H. 1994, A\&A, 287, 145

Siess, L., Dufour, E., \& Forestini, M. 2000, A\&A, 358, 593

Stark, A. A., \& Brand, J. 1989, ApJ, 339, 763

Torres, R. M., Loinard, L., Mioduszewski, A. J., \& Rodríguez, L. F. 2007, ApJ, 671, 1813 
Torres, R. M., Loinard, L., Mioduszewki, A. J., \& Rodríguez, L. F. 2008, Revista Mexicana de Astronomia y Astrofisica Conference Series, 34, 98

Walter, F. M., Brown, A., Mathieu, R. D., Myers, P. C., \& Vrba, F. J. 1988, AJ, 96, 297

Xu, Y., Reid, M. J., Zheng, X. W., \& Menten, K. M. 2006, Science, 311, 54 